\title{
Generalized Fibonacci numbers and Bernoulli polynomials
}

\author{
Anthony G. Shannon ${ }^{1}$, Ömür Deveci ${ }^{2}$ and Özgür Erdağ ${ }^{2}$ \\ ${ }^{1}$ Fellow, Warrane College, The University of New South Wales \\ Kensington NSW 2033, Australia \\ e-mail: tshannon38egmail.com \\ ${ }^{2}$ Department of Mathematics, Faculty of Science and Letters \\ Kafkas University, 36100 Kars, Turkey \\ e-mails: odeveci36@hotmail.com; ozgur_erdag@hotmail.com
}

Received: 25 January 2019

Accepted: 8 March 2019

\begin{abstract}
Relationships, in terms of equations and congruences, are developed between the Bernoulli numbers and arbitrary order generalizations of the ordinary Fibonacci and Lucas numbers. Some of these are direct connections and others are analogous similarities.
\end{abstract}

Keywords: Fibonacci polynomials, Difference operators, Generalized Fibonacci and Lucas numbers, Bernoulli numbers and polynomials.

2010 Mathematics Subject Classification: 11B39, 11B50, 11 B68.

\section{Introduction}

A generating function for Fibonacci polynomials $\left\{u_{n}(x)\right\}[3,4]$ was demonstrated in [3] to be

$$
\sum_{n=0}^{\infty} u_{n}(x) \frac{t^{n}}{n !}=\exp \left(x t+\sum_{m=1}^{\infty} v_{m} \frac{t^{m}}{m}\right)
$$

in which

$$
u_{n}=\left\{\begin{array}{cc}
\sum_{j=1}^{r}(-1)^{j+1} P_{j} u_{n-j}, & n>0, \\
1, & n=0, \\
0, & n<0,
\end{array}\right.
$$


and

$$
v_{n}=\left\{\begin{array}{cc}
\sum_{j=1}^{r}(-1)^{j+1} P_{j} v_{n-j}, & n \geq r, \\
\sum_{j=1}^{r} \alpha_{j}^{n}, & 0 \leq n<r, \\
0, & n<0,
\end{array}\right.
$$

where the $P_{j}$ are arbitrary integers and the $\alpha_{j}$ are the distinct roots of the auxiliary equation

$$
x^{r}=\sum_{j=1}^{r}(-1)^{j+1} P_{j} x^{r-j}
$$

From (1.1) it follows that

$$
u_{n}=\frac{1}{n !} u_{n}(0)
$$

and

$$
\sum_{n=0}^{\infty} u_{n}(x) \frac{t^{n}}{n !}=e^{x t}\left(\sum_{n=0}^{\infty} u_{n}(0) \frac{t^{n}}{n !}\right),
$$

which are comparable with similar results for the Bernoulli polynomials such as

$$
\sum_{n=0}^{\infty} B_{n}(x) \frac{t^{n}}{n !}=e^{x t}\left(\sum_{n=0}^{\infty} B_{n}(0) \frac{t^{n}}{n !}\right) .
$$

Here we prove a more direct connection between these generalized Fibonacci numbers and the Bernoulli numbers, namely

$$
n u_{n-1}(0)=\sum_{k=0}^{n}\left(\begin{array}{l}
n \\
k
\end{array}\right) B_{n-k}(0) \Delta u_{k}(0),
$$

in which the difference operator $\Delta[2]$ is defined as

$$
\Delta u_{n}(x)=u_{n}(x+1)-u_{n}(x) .
$$

\section{Proof of Fibonacci-Bernoulli connection}

The proof of (1.5):

$$
\begin{aligned}
\sum_{n=0}^{\infty} \Delta u_{n}(x) \frac{t^{n}}{n !} & =e^{(x+1) t} \sum_{n=o}^{\infty} u_{n}(0) \frac{t^{n}}{n !}-e^{x t} \sum_{n=0}^{\infty} u_{n}(0) \frac{t^{n}}{n !} \\
& =\left(e^{t}-1\right) e^{x t} \sum_{n=0}^{\infty} u_{n}(0) \frac{t^{n}}{n !}
\end{aligned}
$$

and so

$$
\frac{t}{e^{t}-1} \sum_{n=0}^{\infty} \Delta u_{n}(x) \frac{t^{n}}{n !}=t e^{x t} \sum_{n=0}^{\infty} u_{n}(0) \frac{t^{n}}{n !}
$$


But

$$
\frac{t}{e^{t}-1}=\sum_{n=0}^{\infty} B_{n} \frac{t^{n}}{n !}
$$

so

$$
\begin{aligned}
\frac{t}{e^{t}-1} \sum_{n=0}^{\infty} \Delta u_{n}(x) \frac{t^{n}}{n !} & =\sum_{m=0}^{\infty} B_{m} \frac{t^{m}}{m !} \sum_{n=0}^{\infty} \Delta u_{n}(x) \frac{t^{n}}{n !} \\
& =\sum_{n=0}^{\infty} \sum_{n=0}^{n}\left(\begin{array}{l}
n \\
k
\end{array}\right) \cdot B_{n-k} \Delta u_{k}(x) \frac{t^{n}}{n !}
\end{aligned}
$$

and we also have that

$$
\begin{aligned}
t e^{x t} \sum_{n=0}^{\infty} u_{n}(0) \frac{t^{n}}{n !} & =t \sum_{m=0}^{\infty} x^{m} \frac{t^{m}}{m !} \sum_{n=0}^{\infty} u_{n}(0) \frac{t^{n}}{n !} \\
& =t \sum_{n=0}^{\infty} \sum_{k=0}^{n}\left(\begin{array}{l}
n \\
k
\end{array}\right) u_{n-k}(0) x^{k} \frac{t^{n}}{n !} \\
& =\sum_{n=0}^{\infty} \sum_{k=0}^{n}\left(\begin{array}{l}
n \\
k
\end{array}\right) u_{n-k}(0) x^{k} \frac{t^{n+1}}{n !} \\
& =\sum_{n=0}^{\infty} \sum_{k=0}^{n-1}\left(\begin{array}{c}
n-1 \\
k
\end{array}\right) u_{n-k-1}(0) x^{k} \frac{t^{n}}{(n-1) !}
\end{aligned}
$$

from which we get the required result on equating coefficients of $t^{n}$ when $x=0$.

\section{Fibonacci and Bernoulli congruences}

The Appell set criterion was established in [6], namely,

$$
\frac{d}{d x} u_{n}(x)=n u_{n-1}(x), n=1,2,3, \ldots
$$

from which we can obtain

$$
\int_{x}^{x+1} u_{n}(y) d y=\frac{\Delta u_{n+1}(x)}{n+1},
$$

which parallels the known result [1]

$$
\int_{x}^{x+1} B_{n}(y) d y=\frac{\Delta B_{n+1}(x)}{n+1} .
$$

Just as there are many Bernoulli polynomial congruences [7], so there are too for the Fibonacci polynomials. For instance, we can also show that

$$
u_{n+t n}(x) \equiv u_{n}(x)\left(u_{m}(x)\right)^{t}(\bmod m) .
$$

To prove this, we use induction on $t$ and $m$.

When $t=0$, it is obvious for all $n$.

When $t=1$ and $n=1$, we note that 


$$
u_{1}(x)=x+v_{1}
$$

and

$$
u_{m+1}(x)=\left(x+v_{1}\right) u_{m}(x)+\sum_{j=1}^{m} v_{j+1} m^{-j} u_{m-j}(x),
$$

in which we have used the falling factorial coefficient $m^{-}$[5], and

$$
\sum_{j=1}^{m+s}(m+s)^{\underline{j}} \equiv \sum_{j=1}^{m+s} s^{\underline{j}}(\bmod m) \equiv \sum_{j=1}^{s} s^{\underline{j}},
$$

which we use below. Thus,

$$
\begin{aligned}
u_{m+1}(x) & \equiv\left(x+v_{1}\right) u_{m}(x)(\bmod m) \\
& \equiv u_{1}(x) u_{m}(x)(\bmod m) .
\end{aligned}
$$

Assume the result is true for $n=2,3, \ldots, s$; that is

$$
u_{m+n}(x) \equiv u_{m}(x) u_{n}(x)(\bmod m) .
$$

Then

$$
\begin{aligned}
u_{m+s+1}(x) & =\left(x+v_{1}\right) u_{m+s}(x)+\sum_{j=1}^{m+s} v_{j+1}(m+s)^{-} u_{m+s-j} \\
& \equiv\left(x+v_{1}\right) u_{m+s}(x)+\sum_{j=1}^{s} v_{j+1} s^{-} u_{m+s-j} \quad(\bmod m) \\
& \equiv\left(x+v_{1}\right) u_{m+s}(x)+\sum_{j=1}^{s} v_{j+1} s^{j} u_{s-j} u_{m}(x)(\bmod m) \\
& \equiv\left(x+v_{1}\right) u_{m}(x) u_{s}(x)+\sum_{j=1}^{s} v_{j+1} s^{-} u_{s-j}(x)(\bmod m) \\
& \equiv u_{m}(x)\left(\left(x+v_{1}\right) u_{s}(x)+\sum_{j=1}^{s} v_{j+1} s^{-} u_{s-j}(x)\right)(\bmod m) \\
& \equiv u_{m}(x) u_{s+1}(x)(\bmod m)
\end{aligned}
$$

so when $t=1$, for all $n$

$$
u_{n+m}(x) \equiv u_{n}(x) u_{m}(x)(\bmod m)
$$

and when $t=2$, for all $n$,

$$
u_{n+2 m}(x) \equiv u_{n}(x)\left(u_{m}(x)\right)^{2}(\bmod m) .
$$

Assume the result holds for $t=3,4, \ldots, k$

$$
\begin{aligned}
u_{n+(k+1) m}(x) & \equiv u_{n+k m}(x) u_{m}(x)(\bmod m) \\
& \equiv u_{n}(x)\left(u_{m}(x)\right)^{k+1}(\bmod m) .
\end{aligned}
$$

To continue the Bernoulli connections, it follows that for $n=0,1,2, \ldots$

$$
u_{n}(x)-\left(u_{m}(x)\right)^{t}-u_{n+t m}(x)=\sum_{j=-n}^{t m} B_{j}(n) u_{n+j}(x)
$$

in which the $B_{j}(n)$ are also polynomials depending on $n$ with integral coefficients modulo $m$. 
We then have the following result. Let the monic polynomial elements of the set

$$
\left\{u_{0}(x), u_{1}(x), \ldots, u_{n}(x)\right\}
$$

with coefficients modulo $m$ such that

$$
u_{s}(x)=\sum_{j=0}^{s} a_{s, j} x^{j} \quad\left(a_{s, s}=1,0 \leq s \leq n\right)
$$

and

$$
\sum_{s=0}^{n} A_{s} u_{s}(x) \equiv 0(\bmod m)
$$

where $A_{s}(s=0,1,2, \ldots, n)$ are integral modulo $m$. Then,

$$
A_{s} \equiv 0(\bmod m)(0 \leq s \leq n)
$$

Proof:

$$
\begin{aligned}
\sum_{s=0}^{n} A_{s} u_{s}(x) & =\sum_{s=0}^{n} \sum_{j=0}^{s} A_{s} a_{s, j} x^{j} \\
& \equiv 0(\bmod m) .
\end{aligned}
$$

Now

$$
\sum_{s=0}^{n} \sum_{j=0}^{s} A_{s} a_{s, j} x^{j}=\left[A_{0}, A_{1}, A_{2}, \ldots, A_{n}\right]\left[\begin{array}{ccccc}
a_{0,0} & 0 & 0 & \ldots & 0 \\
a_{1,0} & a_{1,1} & 0 & \ldots & 0 \\
a_{2,0} & a_{2,1} & a_{2,2} & \ldots & 0 \\
& & & \ldots & \\
a_{n, 0} & a_{n, 1} & a_{n, 2} & \ldots & a_{n, n}
\end{array}\right]\left[\begin{array}{c}
1 \\
x \\
x^{2} \\
\ldots \\
x^{n}
\end{array}\right]
$$

so that

$$
\sum_{j=0}^{n} x^{j} \sum_{s=j}^{n} A_{s} a_{s, j} \equiv 0(\bmod m)
$$

which implies that

$$
\sum_{s=j}^{n} A_{s} a_{s, j} \equiv 0(\bmod m),
$$

since if $u_{s}(x)$ is a polynomial with integral coefficients, the statement $u_{s}(x) \equiv 0(\bmod m)$ means that each coefficient of $u_{s}(x)$ is divisible by $m$. Thus, $m \mid A_{s}, s=0,1,2, \ldots, n$, because of the triangularity of the matrix in (3.4). This completes the proof of (3.3).

\section{Concluding comment}

These ideas can be extended to results which further connect the Fibonacci and Bernoulli recurrence relations. 


\section{References}

[1] Carlitz, L. (1965). Recurrences for the Bernoulli and Euler numbers. Mathematische Nachrichten. 2 (3-4), 151-160.

[2] Hartree, D. R. (1958). Numerical Analysis. Oxford: Clarendon Press.

[3] Hoggatt, V. E. Jr, \& Bicknell, M. (1973). Roots of Fibonacci polynomials. The Fibonacci Quarterly. 11 (3), 271-274.

[4] Hoggatt, V. E. Jr, \& Long, C. T. (1974). Divisibility properties of generalized Fibonacci Polynomials. The Fibonacci Quarterly. 12 (2), 113-120.

[5] Knuth, D. E. (1992). Two notes on notation, American Mathematical Monthly, 99 (5), 403-422.

[6] Shannon, A. G. (1975). Fibonacci analogs of the classical polynomials. Mathematics Magazine. 48 (3), 123-130.

[7] Sun, Z.-W. (2003). General congruences for Bernoulli polynomials. Discrete Mathematics. 261 (1-3), 253-276. 